\title{
Vocabulário expressivo de crianças com desenvolvimento fonológico normal e desviante****
}

\author{
Expressive vocabulary of children with normal and deviant \\ phonological development
}

\author{
Marcia de Lima Athayde* \\ Helena Bolli Mota** \\ Carolina Lisbôa Mezzomo**
}

\begin{abstract}
*Fonoaudióloga da Associação de Pais e Amigos dos Excepcionais (APAE) de Santo Ângelo - RS. Mestre em Distúrbios da Comunicação Humana da Universidade Federal de Santa Maria. Endereço para correspondência: Av. Borges de Medeiros, 1900 - Apto. 07 - Santa Maria - RS -

CEP 97015-090

(fga.marcia@yahoo.com.br).
\end{abstract}

**Fonoaudióloga. Doutora em Letras pela Pontifícia Universidade Católica do Rio Grande do Sul (PUCRS). Professora Adjunta do Departamento de Fonoaudiologia da Universidade Federal de Santa Maria.

***Trabalho Realizado na Pós-Graduação em Distúrbios da Comunicação Humana da Universidade Federal de Santa Maria.

Artigo Original de Pesquisa

Artigo Submetido a Avaliação por Pares

Conflito de Interesse: não

Recebido em 16.03.2009.

Revisado em 20.12.2009.

Aceito para Publicação em 22.04.2010.

\begin{abstract}
Background: expressive vocabulary of children with normal and deviant phonological development. Aim: to determine whether alterations presented by children with phonological disorders occur only at the phonological level or if there are any impacts on lexical acquisition; to compare the vocabulary performance of children with phonological disorders to reference values presented by the used test. Method: participants of the study were 36 children of both genders, 14 with phonological disorders (Study group) and 22 with typical language development (Control Group). The ABFW - Vocabulary Test (Befi-Lopes, 2000) was used for assessing the expressive vocabulary of children and later to compare the performance of both groups. Results: the performance of children with phonological disorder in the expressive vocabulary test is similar to that of children with normal phonological development. Most of the children of both groups reached the benchmarks proposed by the test for the different semantic fields. The semantic field Places demonstrated to be the most complex for both groups. Conclusion: the alterations presented by children with phonological disorder area limited to the phonological level, having no impact on the lexical aspect of language.
\end{abstract}

Key Words: Vocabulary; Language Development; Language Development Disorders; Language Tests.

\section{Resumo}

Tema: vocabulário expressivo de crianças com desenvolvimento fonológico normal e desviante. Objetivo: verificar se as alterações das crianças com desvio fonológico ocorrem apenas no nível fonologico ou se há algum impacto na aquisição lexical e comparar o desempenho em vocabulário das crianças desta amostra com os valores de Referência de Normalidade do teste utilizado. Método: a amostra foi composta por 36 crianças de ambos os sexos, sendo 14 com desvio fonológico (Grupo Estudo) e 22 com desenvolvimento normal de linguagem (Grupo Controle). Foi aplicado o teste ABFW - Vocabulário (BefiLopes, 2000) para avaliar o vocabulário expressivo destas crianças e, após, foi comparado o desempenho dos dois grupos. Resultados: o desempenho em vocabulário expressivo das crianças com desvio fonológico é semelhante ao das crianças com desenvolvimento fonológico normal. Grande parte das crianças desta amostra atingiu os valores de referência propostos pelo teste na maioria dos campos conceituais, e o que mais se mostrou complexo para as crianças dos dois grupos foi o Locais. Conclusão: a alteração das crianças com desvio fonológico é realmente apenas a nível fonológico, sem impacto no aspecto lexical da linguagem.

Palavras-Chave: Vocabulário; Desenvolvimento da linguagem; Transtornos do Desenvolvimento da Linguagem; Testes de Linguagem.

Referenciar este material como:

$\checkmark$ Athayde ML, Mota HB, Mezzomo CL. Vocabulário expressivo de crianças com desenvolvimento fonológico normal e desviante. Pró-Fono Revista de Atualização $\sum 3$ Científica. 2010 abr-jun;22(2):145-50. 


\section{Introdução}

Uma das coisas mais importantes que a criança deve aprender é o vocabulário de sua Língua nativa ${ }^{1}$. As crianças normais começam a balbuciar por volta dos 6 aos 9 meses e as primeiras palavras surgem em torno dos 10 aos 15 meses $^{2}$, sendo que as palavras curtas são adquiridas primeiro do que as mais extensas ${ }^{3}$.

Existem crianças que apresentam anormalidades no desenvolvimento fonológico sem uma etiologia orgânica aparente. Estes casos são denominados "desvios fonológicos"4. Esta desordem é caracterizada por uma produção anormal dos sons e uso inadequado das regras fonológicas da Língua, sendo que a causa desta ainda não está definida e sua etiologia é bastante discutida ${ }^{5}$.

Há um forte sincronismo entre o desenvolvimento do léxico e o sistema fonológico. Há crianças com repertório fonético pequeno que tendem a ter relativamente poucas palavras armazenadas no léxico, e crianças com vocabulário amplo e repertório relativamente completo ${ }^{6}$. Se uma criança tem poucos fonemas à disposição e tenta produzir grande número de palavras diferentes, ela simplesmente não consegue, pois não tem sons suficientes para produzir estas palavras, produzindo, então, formas homônimas, o que torna seu discurso difícil de ser compreendido ${ }^{2}$.

Postula-se como hipótese que o vocabulário das crianças com desvio fonológico é semelhante ao das crianças com desenvolvimento normal de linguagem. Desta forma, o objetivo do presente estudo foi o de verificar se as alterações das crianças com desvio fonológico ocorrem apenas no nível fonológico, ou se há algum impacto na aquisição lexical. Ainda, objetiva comparar o desempenho em vocabulário das crianças desta amostra com os valores de referência de normalidade do teste utilizado.

\section{Método}

Este é um estudo de pesquisa realizado a partir de dados clínicos de crianças participantes do projeto de pesquisa "Vocabulário Expressivo e Habilidades de Memória de Trabalho em Crianças com Desenvolvimento Fonológico Normal e Desviante", aprovado no Comitê de Ética em Pesquisa sob número 0102.0.243.000-07.

Os dados foram coletados no período de outubro de 2007 a junho de 2008.

Para os sujeitos fazerem parte da amostra, foram considerados os seguintes critérios de inclusão:
. estarem autorizados pelos pais e/ou responsáveis para a participação na pesquisa por meio da assinatura do Termo de Consentimento Livre e Esclarecido;

- grupo estudo (GE): apresentar diagnóstico de desvio fonológico;

- grupo controle (GC): apresentar adequação nos aspectos compreensivo e expressivo da linguagem, bem como nos seus componentes pragmático, semântico, sintático, morfológico e fonético/ fonológico.

Como critérios de exclusão, consideraram-se os casos de:

. alterações evidentes nos aspectos neurológico, cognitivo e/ou psicológico;

. indícios de perda auditiva;

- alteração do sistema estomatognático que pudessem estar relacionados com as alterações de fala;

. realização de tratamento fonoaudiológico anterior; . não conseguir concluir a avaliação do vocabulário em um dia ou demonstrar dificuldade de concentração.

Foram avaliadas crianças selecionadas a partir das triagens de um serviço público de atendimento fonoaudiológico e de triagens realizadas em duas escolas públicas, sendo uma estadual e a outra filantrópica.

As crianças foram avaliadas individualmente nas próprias instituições de origem e todas passaram por uma triagem fonoaudiológica, na qual foi realizada: avaliação informal da linguagem compreensiva e expressiva por meio de uma sequência lógica; avaliação fonológica completa ${ }^{4}$ para as crianças nas quais foi observada alteração no inventário fonológico; avaliação do sistema estomatognático; e triagem audiológica.

Após, foi aplicada a avaliação de vocabulário do teste ABFW - Teste de Linguagem Infantil ${ }^{7}$, com o objetivo de verificar a competência lexical de cada criança. Por intermédio desta prova, foram avaliados nove campos conceituais: vestuário, animais, alimentos, meios de transporte, móveis e utensílios, profissões, locais, formas e cores, brinquedos e instrumentos musicais. Essa prova analisa as designações por vocábulos usuais (DVU), as não designações (ND) e os processos de substituição (PS) utilizados pelas crianças para alcançar a nomeação correta dos vocábulos. Para cada um dos campos conceituais há uma referência de normalidade $(\mathrm{RN})$. 
TABELA 1. Comparação do desempenho em vocabulário entre os grupos.

\begin{tabular}{|c|c|c|c|c|c|}
\hline Classes & Campos Conceituais & Grupos & Médias & Desvio Padrão & Valor do p \\
\hline \multirow{20}{*}{ DVU } & \multirow[t]{2}{*}{ vestuário } & GE & 79,28 & 12,06 & \multirow[t]{2}{*}{0,8004} \\
\hline & & GC & 79,54 & 12,90 & \\
\hline & \multirow[t]{2}{*}{ animais } & GE & 84,69 & 12,39 & \multirow[t]{2}{*}{0,6971} \\
\hline & & GC & 87,70 & 5,21 & \\
\hline & \multirow[t]{2}{*}{ alimentos } & GE & 70,88 & 14,68 & \multirow[t]{2}{*}{0,0649} \\
\hline & & GC & 79,31 & 7,38 & \\
\hline & meios de & GE & 88,30 & 10,35 & \multirow[t]{2}{*}{0,8390} \\
\hline & transporte & GC & 87,59 & 11,41 & \\
\hline & móveis e & GE & 82,83 & 10,37 & \multirow[t]{2}{*}{0,4375} \\
\hline & utensílios & GC & 82,82 & 7,71 & \\
\hline & \multirow[t]{2}{*}{ profissões } & GE & 44,28 & 16,03 & \multirow[t]{2}{*}{0,1420} \\
\hline & & GC & 52,72 & 16,38 & \\
\hline & \multirow[t]{2}{*}{ locais } & GE & 44,03 & 18,89 & \multirow[t]{2}{*}{0,7435} \\
\hline & & GC & 47,51 & 22,43 & \\
\hline & formas e & GE & 59,28 & 26,44 & \multirow[t]{2}{*}{$0,0322 *$} \\
\hline & cores & GC & 76,36 & 16,48 & \\
\hline & brinquedos e & GE & 67,52 & 19,76 & \multirow[t]{2}{*}{0,0722} \\
\hline & instrumentos musicais & GC & 78,91 & 18,26 & \\
\hline & \multirow[t]{2}{*}{ média } & GE & 69,01 & 10,66 & 0,1047 \\
\hline & & GC & 74,72 & 8,425 & \\
\hline & vestuário & GE & 0,71 & 2,672 & 0,7436 \\
\hline & & GC & 0,45 & 2,132 & \\
\hline & animais & GE & 5,70 & 10,06 & 0,5588 \\
\hline & & GC & 2,42 & 3,87 & \\
\hline & alimentos & GE & 9,30 & 11,60 & 0,2792 \\
\hline & & GC & 4,23 & 4,84 & \\
\hline & meios de & GE & 1,29 & 4,85 & 0,6129 \\
\hline & transporte & GC & 1,23 & 3,19 & \\
\hline & móveis e & GE & 3,56 & 3,20 & 0,0888 \\
\hline $\mathrm{ND}$ & utensílios & GC & 1,89 & 3,07 & \\
\hline IND & profissões & GE & 7,14 & 9,13 & 0,4630 \\
\hline & & GC & 4,54 & 5,95 & \\
\hline & locais & GE & 7,73 & 10,05 & 0,0339* \\
\hline & & GC & 1,89 & 4,40 & \\
\hline & formas e & GE & 10,71 & 13,28 & 0,4197 \\
\hline & cores & GC & 6,81 & 9,94 & \\
\hline & brinquedos e & GE & 9,73 & 9,74 & 0,2218 \\
\hline & instrumentos musicais & GC & 6,61 & 8,95 & \\
\hline & média & GE & 6,21 & 4,81 & 0,0945 \\
\hline & & GC & 3,34 & 2,42 & \\
\hline & vestuário & GE & 19,28 & 11,41 & 0,9727 \\
\hline & & GC & 20,00 & 12,72 & \\
\hline & animais & GE & 9,99 & 11,31 & 0,5450 \\
\hline & & GC & 9,99 & 5,33 & \\
\hline & alimentos & GE & 19,98 & 11,97 & 0,3983 \\
\hline & & GC & 16,04 & 5,68 & \\
\hline & meios de & GE & 10,38 & 7,85 & 0,9191 \\
\hline & transporte & GC & 11,15 & 10,47 & \\
\hline & móveis e & GE & 14,20 & 10,62 & 0,2923 \\
\hline PS & utensílios & GC & 15,28 & 6,69 & \\
\hline & profissões & GE & 48,57 & 21,07 & 0,2629 \\
\hline & & GC & 42,72 & 16,67 & \\
\hline & locais & GE & 48,79 & 16,93 & 0,7190 \\
\hline & & GC & 49,60 & 20,95 & \\
\hline & formas e & GE & 29,28 & 19,79 & 0,0693 \\
\hline & cores & GC & 17,27 & 14,53 & \\
\hline & brinquedos e & GE & 23,37 & 15,42 & $0,0402 *$ \\
\hline & instrumentos musicais & GC & 13,63 & 12,77 & \\
\hline & média & GE & 19,47 & 6,60 & 0,3467 \\
\hline & & GC & 16,99 & 5,93 & \\
\hline
\end{tabular}

Legenda: DVU - designação por vocábulos usuais; ND - não designações; PS processo de substituição; GE - grupo estudo; GC - grupo controle; valores de p significantes $(\mathrm{p}<0,05)$ destacados com asterisco na Tabela; teste estatístico utilizado: Kruskal-Wallis.
As avaliações foram realizadas em três etapas, em dias diferentes. Inicialmente, eram realizadas a triagem fonoaudiológica e a avaliação fonológica; no segundo dia a triagem auditiva; no terceiro a avaliação do vocabulário.

Após a exclusão de 10 crianças, atingiu-se a amostra composta por 36 sujeitos, sendo 14 pertencentes ao GE e 22 ao GC, com idades entre 5:1 e 5:11 anos. A média de idade foi de 5:9 anos para o GE e 5:7 anos para o GC. O GE foi composto por 7 crianças do sexo feminino e 7 do masculino, e o GC por 15 crianças do sexo feminino e 7 do masculino.

Para comparar o desempenho entre os grupos foi utilizado o teste estatístico Kruskal-Wallis e o nível de significância foi fixado em $\mathrm{p}<0,05$. Na parte do estudo que envolveu os valores de $\mathrm{RN}$ foi realizada uma análise qualitativa.

\section{Resultados}

Os dados referentes ao desempenho em prova de vocabulário nos GE e GC são apresentados na Tabela 1.

Em relação às DVU, observou-se que nos campos vestuário, animais, alimentos, profissões, locais, formas e cores, brinquedos e instrumentos musicais e, inclusive, na média de DVU, as crianças do GC apresentaram médias superiores às das crianças do GE. Porém, o GE apresentou médias superiores ao GC nos campos meios de transporte e móveis e utensílios. Apenas a diferença entre os grupos referente ao campo Formas e Cores foi significante estatisticamente.

Quanto às ND, constatou-se que o GE apresentou médias superiores ao GC em todos os campos e, consequentemente, na média das ND. Entretanto, apenas a diferença entre os grupos referente ao campo locais foi estatisticamente significante.

Ao analisar os PS, notou-se que o GE apresentou médias superiores ao GC nos campos alimentos, profissões, formas e cores, brinquedos e instrumentos musicais e na média dos PS, enquanto que o GC apresentou médias superiores ao GE nos campos: vestuário, meios de transporte, móveis e utensílios e locais. No campo alimentos as médias foram iguais. Contudo, apenas a diferença entre os grupos referente ao campo brinquedos e instrumentos musicais foi significante estatisticamente. 
Na Tabela 2 são apresentadas as porcentagens de crianças dos GE e GC que apresentaram resultados satisfatórios em cada campo, de acordo com os valores de RN disponibilizados pelo teste em questão.

Em relação às DVU, pode-se observar que a minoria das crianças do GE e do GC atingiram a normalidade no campo Locais, sendo que o mesmo ocorreu para as crianças do GE também no campo Formas e Cores. Nos demais campos a maioria das crianças atingiu a normalidade.

Quanto às ND, observou-se que nenhum campo mostrou-se complexo para as crianças de ambos os grupos, visto que a maioria apresentou resultados desejáveis em todos os campos.

Na classe dos PS observou-se que a minoria das crianças do GE apresentou resultados satisfatórios nos campos Alimentos e Profissões, sendo que no campo Locais nenhuma criança deste grupo apresentou resultado esperado e no campo Formas e Cores apenas metade das crianças apresentou resultado satisfatório. Quanto ao GC, a minoria das crianças deste grupo apresentou resultados satisfatórios no campo Locais.

\section{Discussão}

Os resultados desta pesquisa, em sua maioria, vão ao encontro da hipótese de que as crianças com desvio fonológico apresentam desempenho em prova de vocabulário semelhante ao das crianças com desenvolvimento fonológico normal, sugerindo que o desvio fonológico afeta apenas o componente fonológico da linguagem.

Comparando-se as DVU entre os GC e GE, notou-se que apenas no campo formas e cores o GC apresenta desempenho significativamente superior ao GE; nos demais campos não houve diferenças estatisticamente significantes.

Em relação às ND, apenas a diferença encontrada no campo locais foi significante, apontando que o GE realizou mais ND neste campo do que o GC; nos demais campos conceituais não houve diferenças estatisticamente significativas.

Quanto aos PS, somente foi estatisticamente significante a diferença entre os grupos no campo brinquedos e instrumentos musicais, no qual as crianças do GE realizaram mais PS do que o GC. Não houve diferenças estatisticamente significativas nos demais campos.

Estes achados vão ao encontro do que era previsto para este estudo, pois sugerem um desempenho em vocabulário semelhante entre os grupos na prova em questão.
TABELA 2. Porcentagens de crianças que apresentaram resultados satisfatórios em cada campo conceitual.

\begin{tabular}{l|c|c|c|c|c|c|c|c|c|l}
\hline Classes & Grupos & Vest & Anim & Alim & MT & MU & Prof & Loc & FC & BI \\
\hline DVU & GE & 85,71 & 100 & 71,42 & 100 & 92,85 & 71,42 & 7,14 & 42,85 & 57,14 \\
& GC & 90,9 & 100 & 90,9 & 100 & 100 & 81,81 & 22,72 & 77,27 & 86,36 \\
\hline \multirow{2}{*}{ ND } & GE & 92,85 & 85,71 & 78,57 & 92,85 & 78,57 & 92,85 & 78,57 & 64,28 & 78,57 \\
& GC & 95,45 & 100 & 100 & 86,36 & 86,36 & 100 & 95,45 & 81,81 & 81,81 \\
\hline \multirow{2}{*}{ PS } & GE & 85,71 & 92,85 & 42,85 & 100 & 100 & 42,85 & 0 & 50 & 71,42 \\
& GC & 90,9 & 100 & 63,63 & 100 & 100 & 59,09 & 13,63 & 72,72 & 86,36 \\
\hline
\end{tabular}

Legenda: valores em porcentagens de crianças que apresentaram resultados satisfatórios; DVU - designações por vocábulos usuais; ND - não designações; PS - processos de substituição; GE - grupo estudo; GC - grupo controle; RN - referência de normalidade; Vest - vestuário; Anim - animais; Alim - alimentos; MT - meios de transporte; MU - móveis e utensílios; Prof - profissões; Loc - locais; FC formas e cores; BI - brinquedos e instrumentos musicais.

Observou-se que grande parte das crianças dos dois grupos apresentou dificuldade no campo locais. Levando-se em consideração que a nomeação de figuras envolve três etapas: identificação do objeto, ativação de seu nome e geração da resposta ${ }^{8}$, as crianças podem ter apresentado baixo desempenho por não reconhecerem as figuras deste campo.

Outra pesquisa ${ }^{9}$ também encontrou que as crianças apresentam desempenho muito ruim no campo locais, o que pode ser justificado pelo input visual fornecido, segundo as autoras.

Uma pesquisa ${ }^{10}$ corrobora esta justificativa ao afirmar que a familiaridade e a frequência dos objetos no cotidiano das crianças é importante para a nomeação, pois estes fatores influenciam na ativação do acesso ao léxico, via memória de curto e longo prazo. Outros autores ${ }^{11}$ também afirmam que a familiaridade e a complexidade visual da figura a ser nomeada são importantes devido ao efeito que causam sobre a memória e outros processos cognitivos. Somado a esses fatores, outra pesquisa ${ }^{12}$ refere que o tempo que a criança leva para nomear a figura (tempo de latência) é mais afetado pelo comprimento do nome da figura do que pela frequência da palavra.

Ainda, o campo formas e cores também mostrou-se mais difícil para a maioria das crianças do GE. Pensa-se que isto pode ser explicado pelo fato de que todas as crianças do GC eram provenientes de pré-escolas, ao contrário das crianças do GE, das quais apenas 9 eram também provenientes de pré-escolas.

Este resultado coincide com o encontrado em outro estudo ${ }^{9}$ no qual as crianças com alterações fonológicas apresentaram vocabulário semelhante ao de crianças com desenvolvimento normal de 
linguagem. Outra pesquisa ${ }^{13}$ constatou resultado semelhante, pois não encontrou diferenças estatisticamente significativas nos três graus de severidade do desvio fonológico estudados (médiomoderado, moderado-severo e severo). As médias de acertos das designações usuais, nãodesignações e processos de substituição, em todos os campos semânticos avaliados foram semelhantes nos três graus de severidade.

Estes achados sugerem que o déficit fonológico não interfere significativamente no desempenho em vocabulário expressivo.

Por outro lado, um estudo longitudinal semelhante $^{14}$ discorda da presente pesquisa ao constatar que o desempenho em vocabulário receptivo de indivíduos com alteração de fala foi significativamente menor do que o desempenho do GC na idade de 5 anos.

Ao comparar o desempenho dos grupos com os valores de RN da prova em questão, observouse que, em relação às DVU, grande parte das crianças dos dois grupos apresentou dificuldades no campo locais, sendo que a maioria das crianças do GE também apresentou dificuldades no campo formas e cores. A maioria das crianças não apresentou dificuldade nos demais campos conceituais da prova.

A maioria das crianças dos dois grupos não apresentou dificuldade em nenhum campo conceitual, no que se refere à classe das ND. Em relação aos PS, grande parte das crianças do GE apresentou dificuldade nos campos alimentos, profissões e locais, sendo que no campo formas e cores, metade das crianças deste grupo apresentou

\section{Referências Bibliográficas}

1. Gathercole SE, Baddeley AD. Evaluation of the role of phonological STM in the development of vocabulary in children: A longitudinal study. Journal of Memory and Language. 1989;28:200-13.

2. Stoel-Gammon C. Normal and disordered phonology in two-years-olds. Topic in language disorders. 1991;11(4): 21-32.

3. Maekawa J, Storkel HL. Individual diferences in the influence of phonological characteristics on expressive vocabulary development. J. Child Lang. 2006;33:439-59.

4. Yavas M, Hernandorena CL, Lamprecht RR. Avaliação fonológica da criança: reeducação e terapia. Porto Alegre: Artmed Editora; 2001. dificuldade. Ainda, a maioria das crianças do GC também apresentou dificuldades no campo locais. Grande parte das crianças alcançou o resultado esperado nos demais campos conceituais.

Este resultado sugere que as crianças dos dois grupos estudados preferem substituir a palavraalvo do que não designarem. Uma pesquisa ${ }^{10}$ discorda deste achado ao constatar que crianças mais velhas (5 e 6 anos) referem não saber nomear a figura quando não a reconhecem, ao invés de cometerem mais erros.

As palavras referentes a objetos são produzidas com mais precisão do que aquelas referentes a ações, pois os referentes das palavras para objetos são estáveis e concretos. Ao contrário, os referentes das palavras de ações são transitórios ${ }^{4}$. Isto talvez justifique a dificuldade que as crianças apresentaram no campo conceitual profissões, cujas figuras representam ações.

\section{Conclusão}

O desempenho em vocabulário expressivo das crianças com desvio fonológico é semelhante ao das crianças com desenvolvimento fonológico normal, o que confirma a hipótese de que a alteração das crianças com desvio fonológico é realmente apenas em nível fonológico, sem impacto no aspecto lexical da linguagem.

Constata-se também que grande parte das crianças desta amostra atingiu os valores de referência propostos pelo Teste, na maioria dos campos conceituais, e o campo que mais se mostrou complexo para as crianças dos dois grupos foi 0 locais.

5. Wertzner HF, Amaro L, Galea DES. Phonological performance measured by speech severity indices compared with correlated factors. Sao Paulo Med J. 2007;125(6): 309-14.

6. Stoel-Gammon C. Issues in phonological development and disorders. In: Miller J. (Ed.). Research on child language disorders. Austin, TX: Pro-Ed; 1990.

7. Befi-Lopes DM. Vocabulário. In: Andrade CRF, BefiLopes DM, Fernandes FDM, Wertzner HF. Teste de linguagem infantil: nas áreas de fonologia, vocabulário, fluência e pragmática. Carapicuíba: Pró-Fono; 2000.

8. Paivio A, Clark JM, Digdon N, Bons T. Referential processing: Reciprocity and correlates of naming and imaging. Memory and Cognition. 1989;17(2):163-74. 
9. Befi-Lopes DM, Gândara JP. Desempenho em prova de vocabulário de crianças com diagnóstico de alteração fonológica. Rev. da Soc. Bras. de Fonoaudiologia. 2002; ano $7(1)$ : 16-23.

10. Scheuer CI, Stivanin L, Mangilli LD. Nomeação de figures e a memória em crianças: efeitos fonológicos e semânticos. Pró-Fono Rev Atual Científica. 2004; 16(1): 49-56.

11. Cycowicz YM, Friedman D, Rothstein M, Snodgrass JG. Picture naming by young children: norms for name, agreement, familiarity, and visual complexity. J Exp Child Psychol. 1997;65:171-237.
12. Morrisson CM, Ellis AW, Quinlan PT. Age of acquisition, not word frequency, affect object naming, not object recognition. Memory and Cognition. 1992;20(6):705-14.

13. Pereira LF. Desvio fonológico: desempenho de préescolares em tarefas linguisticas e metalinguísticas nos diferentes graus de gravidade. [tese]. São Paulo: Universidade Federal de São Paulo - Escola Paulista de Medicina; 2006.

14. Beitchman JH, Jiang H, Koyama E, Johnson CJ, Escobar M, Atkinson L, Brownlie EB, Vida R. Models and determinants of vocabulary growth from kindergarten to adulthood. J Child Psychol Psychiatry. 2008;49(6):626-34. 